\title{
Angiogenic zip code
}

\author{
Judah Folkman
}

Good things often come in small packages. In this issue, Renata Pasqualini and colleagues ${ }^{1}$ have used phage panning to identify a novel peptide, only 10 residues in length, that not only selectively targets angiogenic blood vessels but also inhibits the matrix metalloproteinases (MMPs) MMP-2 and MMP-9. When injected into mice, this peptide effectively impedes both growth and invasion of established tumors. Ultimately, therapeutics based on this peptide could prove useful alternatives to MMP inhibitors currently in trials as anticancer agents.

Before the early 1980s, it was generally thought that endothelial cells, and those of the microvascular endothelia in particular, were homogenous. Only in 1981, when Auerbach ${ }^{2}$ showed that the endothelia in different organs express organ-specific antigens, did the heterogeneity of microvascular endothelia begin to be fully appreciated. Further studies have supported the heterogeneity of endothelial morphology and function, even in the early embryo before the
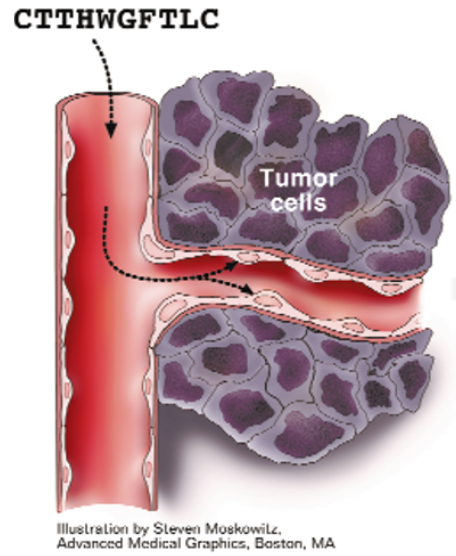

Figure 1. Diagram of the effect of the cyclic peptide CTTHWGFTLC on two cell populations, tumor endothelium and tumor cells. growing blood vessels of mouse tumors. Pasqualini and Ruoslahti subsequently took advantage of their tumor endothelium-targeting peptide to address a conventional cytotoxic chemotherapeutic agent to a murine tumor ${ }^{5}$. This targeting allowed them to achieve antitumor efficacy at significantly lower doses, thereby reducing the drug's toxicity to normal tissues.

In the present paper, Pasqualini and colleagues advance their technology one step further-and it is a major step. Using phage to active endothelial cells but not to quiescent endothelial cells in the vasculature. As the authors emphasize, Cheresh and colleagues ${ }^{6}$ have proposed a mechanism for this anchorage based on the complexing of MMP-2 with $\alpha_{v} \beta_{3}$ integrin, which is itself upregulated on migrating and proliferating endothelial cells.

Numerous potential applications could be envisioned for the newly discovered cyclic decapeptides. If antiangiogenic proteins such as thrombospondin ${ }^{7}$, angiostatin ${ }^{8}$, endostatin $^{9}$, or pigment epithelium-derived factor ${ }^{10}$ could be grafted onto CTTHWGFTLC in such a way that its affinity for MMP-2 and MMP-9 is preserved in the chimera, these inhibitors might be effective at smaller doses. Other possibilities include enhancing magnetic resonance imaging of tumors, decreasing side effects of conventional chemotherapeutic agents, targeting brain tumors with drugs or proteins that do not cross the blood-brain barrier, heart begins beating. For instance, Wang et al. ${ }^{3}$ recently reported that in mice, a membrane-bound ligand of the ephrin family, Eph-B2, marks future arterial but not venous endothelial cells, whereas one of the receptors for Eph-B2 (i.e., Eph-B4) reciprocally marks the venous endothelium.

In 1997, Pasqualini et al. ${ }^{4}$, in Erkki Ruoslahti's laboratory at the Burnham Institute in La Jolla, California, published a provocative paper showing that angiogenic endothelial cells in the vascular bed of a tumor could be distinguished from nongrowing endothelial cells elsewhere in the vasculature by intravenously injecting a peptide discovered by in vivo phage display. This specificity was mediated by a nine-residue cyclic peptide containing an ArgGlyAsp sequence (RGD), which bound with high affinity to alpha integrins expressed in the

Judah Folkman is professor in Departments of Cell Biology and Pediatric Surgery, Harvard Medical School and Children's Hospital, Boston, MA 02115. display technology, they identified a novel cyclic decapeptide, CysThrThrHisTrpGlyPheThrLeuCys (CTTHWGFTLC), which has three functions wrapped up in one package. These functions are the following: first, selective and potent inhibition of MMP2 (gelatinase A) and MMP-9 (gelatinase B), but not of several other MMP family members; second, inhibition of migration of human tumor cells and endothelial cells; and third, targeting to angiogenic vessels (see Fig. 1). Thus, the same peptide that contains the information to locate an angiogenic tumor bed also carries specific antiprotease activity that contributes to a delay in the appearance of tumors, significant slowing of tumor growth, and prolongation of survival in tumor-bearing mice.

Interestingly, this study began with a search for binders of the metalloproteinases MMP-2 and MMP-9, and ended in the discovery of a novel class of cyclic peptides containing an HWGF motif. The high affinity of this motif for the proliferating endothelium in an angiogenic site suggests that MMP-2 and MMP-9 must be preferentially anchored delivering antivascular therapies that coagulate tumor blood vessels ${ }^{11}$, and targeting angiogenesis inhibitors to angiogenic sites in the eye or in atherosclerotic plaques ${ }^{12}$, or in endometriosis ${ }^{13}$.

If the cyclic peptide is successful in even a small fraction of these possible applications, it will certainly package a powerful punch in the fight against cancer and expanding our knowledge of cancer mechanisms. The pharmacology of angiogenic sites is now an open field and \footnotetext{
1. Koivunen, E. et al. Nat. Biotechnol. 17, 768-774
(1999).

2. Auerbach, R. Inter. J. Rad. Biol. 60, 1-10 (1991).

3. Wang, U.H., Chen, Z. \& Anderson, D.J. Cell. 93, 741-753. (1998)

4. Pasqualini, R., Koivunen, E. \& Ruoslahti, E. Nat. Biotechnol. 15, 542-546 (1997).

5. Arap, W., Pasqualini, R. \& Ruoslahti, E. Science. 279, 377-380 (1998)

6. Brooks, P.C. et al. Cell. 5; 683-693 (1996).

7. Dawson, D.W. et al. Mol. Pharmacol. 55, 332-338 (1999).

8. O'Reilly, M.S. et al. Cell. 79, 315-328 (1994).

9. O'Reilly, M.S. et al. Cell. 88, 277-285 (1997).

10. Dawson, D.W. et al. Science. 285, 245-248 (1999).

11. Huang, $X$. et al. Science. 275, 547-550 (1997).

12. Moulton, K.S. et al. Circulation. 99, 1726-1732 (1999).

13. Donnez, J. et al. Hum. Reprod. 13, 1686-1690 (1998).
} it is exciting to think about its future. 